\title{
TADAM: A dynamic whole-stand approximation for the TASS growth model
}

\author{
by Oscar García ${ }^{1}$
}

\section{Errata}

The Forestry Chronicle 81(4): 575-581, 2005.

Ignore material that was originally printed in square brackets in the caption to Figure 5. Corrected caption below.

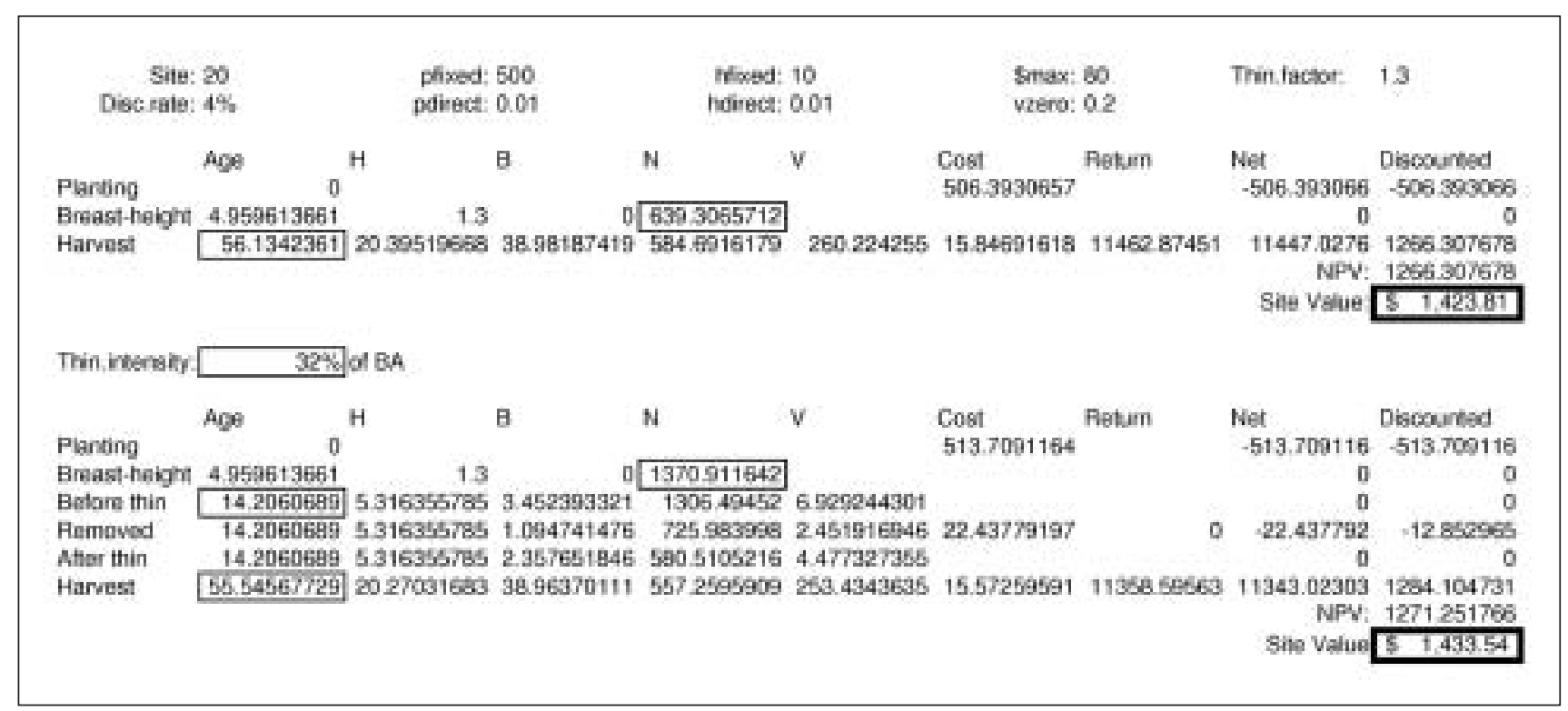

Fig. 5. A spreadsheet using the TADAM Excel add-in to optimize stand treatments.

Missing text on page 580 is indicated in bold:

\section{Closing Remarks}

Detailed mechanistic individual-based models are invaluable as research tools. They can synthesize and make sense of isolated facts, identify knowledge gaps, and generate hypotheses for further investigation. In addition, TASS was able to produce plausible predictions at a time when little permanent sample plot data was available. For the management of evenaged single-species stands, however, whole-stand models based on aggregate stand-level variables can be more useful and potentially more accurate (e. g., Vanclay 1994). Besides issues of complexity, usability, and overparameterization, detailed initial information at the tree level may be unavailable or unreliable, and largely redundant.

TADAM has been able to approximate closely stand dynamics as simulated by TASS. In the longer term, it seems natural to develop similar models from actual field measurements. Meanwhile, TADAM complements TASS and TIPSY as an easy to use and flexible analysis tool. 